Revue de l'Institut des langues et cultures

d'Europe, Amérique, Afrique, Asie et Australie

22 | 2015

La révolution théâtrale dans le Río de la Plata

\title{
Copi et Puig, ovnis du théâtre argentin ?
}

Copi and Puig: UFOs of Argentinian Theatre?

\section{Lionel Souquet}

\section{OpenEdition}

Journals

Édition électronique

URL : http://journals.openedition.org/ilcea/3242

DOI : $10.4000 /$ ilcea.3242

ISSN : 2101-0609

Éditeur

UGA Éditions/Université Grenoble Alpes

Édition imprimée

ISBN : 978-2-84310-295-0

ISSN : 1639-6073

\section{Référence électronique}

Lionel Souquet, « Copi et Puig, ovnis du théâtre argentin ? », ILCEA [En ligne], 22 | 2015, mis en ligne le 01 mars 2015, consulté le 01 mai 2019. URL : http://journals.openedition.org/ilcea/3242 ; DOI :

10.4000/ilcea.3242

Ce document a été généré automatiquement le 1 mai 2019.

(C) ILCEA 


\section{Copi et Puig, ovnis du théâtre argentin?}

Copi and Puig: UFOs of Argentinian Theatre?

Lionel Souquet

1 Malgré l'avant-gardisme de nombreux dramaturges latino-américains, le public argentin restera longtemps frileux, voire conformiste, comme le souligne Stéphanie Urdician (2006: 245-258) : jusqu'au milieu des années 1960, la scène est dominée par un théâtre réaliste, héritier du costumbrismo. Urdician rappelle qu'à cette époque «[...] se confirme une stratégie de contrôle déjà mise en œuvre par le péronisme ainsi que par les guides de la "Révolution libératrice" " (2009: 14) et cite un commentaire d'Andrés Avellaneda (1993 : 13-15) qui résume parfaitement la mainmise croissante du pouvoir sur toute la vie intellectuelle et culturelle, jusqu'à la fin de la dictature, en 1983 :

Les coups d'État de 1962-1964 et 1966-1973 sont une étape d'accumulation pour la législation répressive, qui reprend d'importantes contributions antérieures en matière de censure (surtout des premiers gouvernements de la Révolution libératrice, 1955-1958, et d'Arturo Frondizi, 1958-1962). Vers 1975 les éléments de base du discours de censure sont déjà posés [...] Ce qui n'est pas moral comprend trois grandes catégories : a) l'obscénité : l'idée de sexe comme absence de décence ; l'homosexualité ; la prostitution, la non-famille (adultère, avortement, désamour filial, dégradation du mariage) ; b) l'offense ou l'attaque à l'égard des institutions religieuses, de l'Église catholique ou de la morale chrétienne; c) les agressions contre la sécurité et l'intérêt national. (Urdician, 2009 : 14-15. Je traduis.)

C'est le metteur en scène Jorge Petraglia qui réussira - avec l'aide de l'Institut Torcuato Di Tella - à faire découvrir, dans les années soixante, des auteurs dramatiques tels que Cocteau, Beckett, Adamov ou Pinter. Mettant en scène la désintégration des relations familiales, El desatino, première pièce de Griselda Gambaro (qui deviendra l'une des plus importantes dramaturges argentines), jouée à Buenos Aires en 1965 (pendant la brève accalmie politique de la présidence d'Illía), va créer une polémique parmi le public et les dramaturges « réalistes » alors qu'elle avait été épargnée par la censure. En réponse à la réalité argentine, Gambaro - nous explique Urdician - crée un théâtre physiquement, linguistiquement et symboliquement violent. Proche du concept de «cruauté » d'Artaud, 
Gambaro applique aussi la distanciation brechtienne pour une démystification de tous les poncifs. L'humour est son principal procédé distanciateur et la deviatio le fil d'Ariane de son écriture (Urdician, 2009).

Malgré la dictature du général Onganía (1966-1970) et sa répression des milieux intellectuels (Noche de los Bastones Largos), malgré la Loi de Censure cinématographique, les œuvres placées sous séquestre, Alan Pauls (1986: 11-16) décrit la fin des années soixante comme une période de résistance intellectuelle et artistique et, donc, de créativité et d'ébullition culturelle. C'est à cette époque, en 1968, que Copi (Raúl Damonte Botana, 1939-1987) publie sa première pièce, La journée d'une rêveuse puis, un an après, Eva Peron. Il est cependant difficile de mesurer - et indispensable de relativiser - l'influence du théâtre latino-américain et surtout rioplatense sur son œuvre dramatique. Par son milieu familial, Copi a baigné dès l'enfance dans la vie théâtrale internationale, mais il ne découvre Buenos Aires qu'en 1955 (chute de Perón), à 16 ans, et retourne vite à Paris où il passe son bac et où il s'installe définitivement en 1962, après le coup d'État contre Frondizi. On peut supposer que Copi lit les pièces des auteurs latino-américains mais, lorsqu'il devient dramaturge, il est coupé de la réalité de la scène argentine. Il écrit d'ailleurs ses premières pièces, comme presque toute son œuvre (Barège, 2013 ; Souquet, 2013), en français (Cachafaz ou La sombra de Wenceslao sont de rares exceptions) : il va même jusqu'à franciser le nom des «Perón" en lui enlevant son accent! Marcial Di Fonzo Bo, qui a mis en scène et interprété (comme son oncle Facundo Bo, avant lui) de nombreuses pièces de Copi, explique : «Quand j'étais en Argentine, je ne connaissais pas Copi. Ses œuvres n'étaient pas publiées pendant la dictature de la junte militaire et les quelques années qui ont suivi. » (Celik, 2006 : 61-64) C'est donc en arrivant à Paris, en 1987 (quelques jours seulement avant la mort de Copi), qu'il découvre réellement son œuvre, notamment en assistant Alfredo Arias. Mais pour Jorge Dubatti, critique et historien du théâtre argentin, il est évident que Copi reste un auteur argentin bien que les conventions et l'imaginaire de son théâtre viennent de la post-avant-garde française : « ¿Son argentinos la ferocidad, el absurdo, la violencia, el deseo, el desenmascaramiento, el canibalismo de las piezas teatrales de Copi? Por supuesto ${ }^{1}$ » (Dubatti, 2002) Copi confirme luimême cette idée, lors d'un entretien accordé à Raquel Linenberg-Fressard :

R.L. : Dans une interview que tu as donnée à la revue Masques (Joecker, 1981 : 33-38) tu as dit que même si tu écrivais en français ou en d'autres langues, tu étais un auteur argentin. Alors, on peut toujours être un auteur argentin même si on écrit en français...

Copi : Oui, parce que de toutes façons [...] l'on apprend à penser et à s'exprimer lorsqu'on est tout petit, de sorte que les mots que l'on utilise appartiennent au monde de l'enfance. [...] On ne peut pas dire que je sois un écrivain français, je suis un écrivain argentin, de toute évidence... (Linenberg-Fressard, 1987 : 158-159)

On ne saurait oublier que la grand-mère de Copi, Salvadora Medina Onrubia de Botana (1894-1972), grande bourgeoise et anarcho-féministe, fut une dramaturge reconnue. Elle s'intéresse au théâtre par vocation artistique mais aussi pour des motifs politiques. Sa pièce Alma fuerte (1913) est la première œuvre théâtrale anarchiste écrite par une femme dans le Río de la Plata. Salvadora connaîtra ensuite, à Buenos Aires, une brillante carrière d'auteure dramatique que la culture officielle tentera plus tard d'étouffer et d'effacer. Las descentradas (1928), œuvre clef de l'anarcho-féminisme, sera acclamée par la critique. Cristina Guzzo (2004) montre que Salvadora Medina désarticule, ici, l'utopie libertaire en analysant la femme comme sujet social et individuel. Elle transforme ainsi la doctrine en pratique libératrice et individuelle. Nous verrons que cette alliance entre sujet social et 
individuel - entre politique et questionnement intime et métaphysique - se retrouvera chez Copi, sous une forme certes non réaliste et plus cryptée.

5 Manuel Puig (1932-1990), romancier déjà reconnu dès 1968, viendra au théâtre presque par hasard, dix ans après Copi, en 1978, avec l'adaptation scénique de El beso de la mujer araña (créée en 1981). Jusqu'à ses vingt-quatre ans, Puig vit en Argentine mais, tout à sa passion du cinéma, il est encore relativement éloigné de la vie littéraire et, notamment, théâtrale. De 1956 à 1967, il vit dans plusieurs pays d'Europe puis à New York où il termine son premier roman. Le retour en Argentine dure à peine six ans car, en 1973, la Triple $\mathrm{A}$, la police secrète d'extrême droite, le pousse à prendre le chemin (définitif) de l'exil (Mexique, New York, Rio de Janeiro et, de nouveau, le Mexique où il meurt). L'adaptation à la scène de $E l$ Beso de la mujer araña, un roman sur le cinéma, était un défi extraordinaire qui impliquait des dilemmes presque cornéliens car, pour des raisons techniques évidentes, il fallait procéder à des coupes sombres afin de réduire l'œuvre. Finalement, malgré un bilan un peu mitigé, Puig s'estimera relativement satisfait du résultat : après avoir conservé un seul des six récits de films (Cat people) et éliminé $25 \% \mathrm{du}$ dialogue original, il obtint des répliques plus vivantes et "naturelles » mais moins ambiguës et, donc, plus superficielles.

6 Cette expérience au bilan mitigé pouvait laisser penser que l'écriture dramatique n'était qu'un épiphénomène dans l'œuvre du romancier cinéphile. Cependant, Bajo un manto de estrellas, en 1981, puis la publication posthume de cinq autres pièces et comédies musicales - El misterio del ramo de rosas, Triste golondrina macho, Amor del bueno (Melodrama) , Muy señor mío (Comedia musical), Gardel, uma lembrança (Comedia musical) - montreront que Puig portait un intérêt réel à l'écriture dramatique (Capellán, 1995 : 163-176). En 1999, Nadine Dejong (1999 : 8-17) battait d'ailleurs en brèche l'idée (fort répandue chez les universitaires et relayée par les journalistes littéraires) selon laquelle le septième art aurait été - pratiquement - l'unique source d'inspiration thématique, esthétique et scripturale de Puig. Il est vrai que cette thèse qui, comme nous l'avons vu, est loin d'être sans fondements, a constamment été confirmée par l'auteur lui-même. Cependant, Dejong cite une interview dans laquelle Puig déclare avoir trouvé dans le "Teleteatro" (pièces télévisées) une nouvelle source d'inspiration ${ }^{2}$. S'appuyant sur une analyse très concrète du roman $E l$ beso..., Dejong démontre que Puig: « [...] tourne le dos à l'illusion référentielle immédiate du cinéma et s'inscrit dans une pratique de l'écriture tout à fait théâtrale »(Dejong, 1999 : 16).

\section{Bajo un manto de estrellas de Puig : un théâtre hallucinatoire}

7 Bajo un manto de estrellas (créée en 1982, au Teatro Ipanema de Rio de Janeiro, avec une mise en scène d'Ivan de Albuquerque) est une pièce dont la trame, assez complexe, nous fait louvoyer sans cesse entre rêve et réalité, dans un jeu de miroirs et d'apparences. Puig a rarement porté si loin l'ambiguïté des personnages et des situations.

8 L'action se situe en 1948, dans une luxueuse maison de la bourgeoisie rurale. À la suite de la mort de leurs amis intimes dans un accident de voiture, en 1929, les propriétaires de la maison (un couple d'une cinquantaine d'années : DUEÑo DE CASA / MAÎTRE DE MAISON et D ueÑa DE CASA / MAîtresSe de MAison) ont adopté la fille (Hija / Fille) de ceux-ci. Mais ils s'inquiètent pour la santé mentale de la belle jeune fille qu'elle est devenue. Celle-ci 
informe sa mère adoptive - en cachette du père - que son fiancé, Antonio (le seul personnage qui porte un nom), l'abandonne pour en épouser une autre, plus riche. Sur ces entrefaites, arrive un mystérieux couple d'une trentaine d'années (LA VISITANTE / LA V ISITEUSE et EL VISITANTE / LE VISITEUR ${ }^{3}$ ), en très élégante tenue de soirée, mais à la mode de 1929. Ils prétendent être tombés en panne d'essence alors qu'ils se rendaient à un bal masqué. En fait, il s'agit de deux rats d'hôtel en fuite après avoir dérobé des bijoux, mais nous comprenons, peu à peu, que les propriétaires ont cru reconnaître en eux les parents - défunts - de la jeune fille. À la fois par précaution et par malice, les visiteurs se mettent à jouer le jeu. Profitant alors d'un moment d'intimité, la propriétaire fait une déclaration passionnée au visiteur comme s'il était l'ancien amant dont elle a parlé à sa fille adoptive. Elle prend ensuite congé mais, à peine seul, le visiteur est rejoint par la jeune fille qui, à son tour, lui fait une déclaration enflammée : elle croit reconnaître en lui Antonio, son fiancé. L'homme est très excité par la situation et voit là une opportunité de profiter de la superbe jeune fille qui s'offre à lui. Ils sont alors surpris dans leurs ébats par les maîtres de maison et par la compagne du visiteur. Mais le père craignant encore pour l'équilibre mental de sa fille adoptive - n'oublions pas qu'à ses yeux elle est en train de coucher avec son propre père, son « vrai » père -, il lui fait croire qu'ils ne sont tous les trois qu'une hallucination:

DuEÑo DE CASA. - (Apiadándose, siempre pendiente de lo que para él es la frágil salud mental de la Hija.) No te inquietes [...] Cuando una doncella es desflorada siempre ocurre lo mismo, ella se imagina que los padres la descubren [...] somos una alucinación. Es tu conciencia culpable que te hace ver visiones [...]. (Puig, $1983: 34$ )

$\mathrm{Au}$ début du deuxième acte, le propriétaire décide de tendre un piège à sa femme et à sa fille adoptive : il leur fait croire qu'il ne lui reste plus que quelques mois à vivre, car il est atteint d'un mal étrange et incurable. Au fond, sa femme est contente parce qu'elle pourra fuir avec le visiteur qu'elle croit être son amour retrouvé. Sa fille lui déclare qu'il continuera à vivre en elle comme tous les êtres que l'on aime et qu'en souvenir de lui elle vivra follement son amour avec Antonio. Sa mère adoptive lui apprend alors que les visiteurs ne sont autres que ses vrais parents et la visiteuse, qui n'en croit pas un mot, joue le jeu dans l'espoir de récupérer son compagnon. Les visiteurs décident ensuite de fuir en prenant la fille en otage pour que les propriétaires n'appellent pas la police. La propriétaire apparaît alors avec l'arme des visiteurs et, par dépit, elle tue l'homme, puis la femme. La jeune fille tente alors de se suicider mais son père adoptif l'en empêche. Ce dernier perd soudain son flegme et son air de supériorité en déclarant que sa femme est la seule coupable : "[...] es ella la única culpable, primero esposa adúltera y ahora asesina ${ }^{5} . .$. » (Puig, 1983 : 53). Il se déclare, en fait, amoureux de sa fille adoptive et veut fuir avec elle. C'est alors qu'arrivent un médecin et une infirmière, joués par les mêmes acteurs qui tenaient le rôle des visiteurs (au tout début de la pièce, alarmé par le retard de sa fille, le propriétaire avait en effet appelé l'hôpital psychiatrique). Obéissant aux souhaits des propriétaires, le médecin et son infirmière emmènent la fille. Restés seuls, les parents adoptifs se lamentent et la femme répète la phrase déjà prononcée par elle et par sa fille : "Era demasiada la felicidad que ella nos daba, no la soportamos, solos empezamos a imaginar obstáculos, de ahí a materializarlos el paso fue corto ${ }^{6}$. (Puig, 1983 : 59) La nouvelle bonne, que l'on attendait depuis le début de la pièce, arrive au moment où ils sont sur le point de se suicider (le rôle est tenu par l'actrice qui jouait la fille). Les propriétaires acceptent qu'elle reste et finissent même par se convaincre que c'est leur fille adoptive qui est revenue et que rien ne s'est passé. Finalement, effrayée par les paroles et le comportement des maîtres de maison, la bonne s'enfuit en emportant les bijoux (le butin 
des visiteurs) qu'elle vient de découvrir. Le couple n'a rien vu, il est maintenant heureux, en paix. La femme avoue qu'elle espère toujours le retour de son amant, mais son mari ne s'en sent que plus amoureux : «Si él vuelve por ti, eso significa que vales [...] $]^{7}$ (Puig, 1983 : 65). On entend la sirène de la police, ils s'enlacent, le rideau tombe ${ }^{8}$.

\section{Acteurs en quête de rôles et personnages en quête d'identité : le drame « schizo »}

Dans une analyse magistrale, Milagros Ezquerro (1983) montre comment Puig, par tout un ensemble de mécanismes, parvient à jeter un doute sur l'identité «réelle » (au sein de l'intrigue) des personnages. Afin de complexifier l'écheveau des méprises et des (fausses ? ) reconnaissances, l'auteur a pris le parti de démultiplier les personnages en attribuant huit rôles à cinq comédiens : ceux qui jouent les visiteurs doivent également interpréter le médecin et l'infirmière ; l'actrice qui tient le rôle de la fille incarne ensuite la bonne. En outre, les personnages jouent eux-mêmes des rôles différents selon les destinataires: le visiteur, par exemple, est un voleur de bijoux pour le spectateur alors que le maitre de maison croit y reconnaître le père de la jeune fille et que celle-ci y voit son ex fiancé, Antonio ! Comme le note M. Ezquerro, seul le spectateur est capable de faire la synthèse. Exploitant pleinement ce potentiel dramaturgique de la représentation, absent du système romanesque, Puig déstabilise les spectateurs en les plongeant dans un univers où les certitudes et la logique se dérobent en permanence. Tout contribue à rendre les personnages ambigus et à alimenter les doutes du public : aucun personnage présent sur scène n'a de nom et, selon les indications de l'auteur, les maitres de maison sont « d'âge indéterminé, on dirait la cinquantaine » et les visiteurs " [...] son jóvenes, de unos treinta años, pero al mismo tiempo su aire mundano los torna mayores, de edad indefinida ${ }^{9}$ »(Puig, 1983 : 19). La tenue anachronique des visiteurs, à la mode de 1929 alors que l'action se situe en 1948, ajoute encore à l'ambiguïté des personnages et alimente les doutes du spectateur. Toutes les indications scéniques tendent «[...] à problématiser l'identification des personnages, à rendre leur identité aléatoire, flottante " (Ezquerro, 1983: 50). Les visiteurs sont-ils des voleurs arrivés par hasard ? Pourraient-ils être les vrais parents de la jeune fille? Dans ce cas, seraient-ils toujours vivants alors qu'on les croyait morts ou bien ont-ils ressuscité, ce qui impliquerait que l'œuvre s'inscrive dans un cadre irrationnel et fantastique? Sont-ils une hallucination? Certains personnages sont-ils fous ? Si oui, lesquels ?... Petit à petit, le spectateur glane des indices - notamment le sac de bijoux servant d'objet-témoin - qui lui permettent d'établir son propre « diagnostic ».

Bajo... est une œuvre aussi riche que déconcertante où tout est poussé à son paroxysme par le biais de personnages sur le fil du rasoir. Certains, les propriétaires et leur fille adoptive, sont effectivement passés de l'autre côté du miroir, ils sont vraiment fous, mais «les visiteurs" flirtent, eux aussi, avec la folie. Et c'est le plus intéressant: cette confrontation entre des personnages qui, aux yeux du spectateur et donc de la société, sont indubitablement fous et d'autres que l'on ne considérera pas comme tels. En fait, les personnages sains, les visiteurs, sont plus « pervers » que les propriétaires et leur fille, les «vrais» fous. Si les visiteurs acceptent immédiatement d'entrer dans le jeu des propriétaires, ce n'est pas uniquement parce qu'ils veulent se cacher : c'est aussi parce que la situation les excite par son ambiguïté et par le pouvoir qu'elle leur donne sur leurs hôtes. Mais les visiteurs entrent dans le jeu de rôles comme on entre dans des sables mouvants. Ils sont rapidement happés, dépassés par leurs personnages et 
inéluctablement entraînés vers un dénouement fatal (dans son scénario La Cara del Villano Puig joue avec la même ambiguïté : on se demande constamment qui est sain et qui est fou....). Nous allons voir que la figure du fou libère une écriture du retournement.

Respectant strictement la convention réaliste du "quatrième mur ", qui interdit aux personnages de s'adresser au public, mais sollicitant le «diagnostic» des spectateurs - «diagnostic» sur les personnages qui s'oppose radicalement à l'«expertise " benjaminienne sur les acteurs -, Puig interroge la notion même de distanciation. Jonglant avec les conventions théâtrales, le dramaturge met en place un système si ambigu qu'il oblige le public à s'interroger sur le réalisme de la situation représentée. M. Ezquerro souligne l'une des consignes auctoriales : «Nada es realista, todo es estilizado ${ }^{10}$. » (Puig, 1983: 11) Cependant, Puig joue encore de cette stylisation qu'il nuance constamment de réalisme afin de maintenir l'ambiguïté. Les éléments réalistes sont nombreux: l'intrigue repose sur les relations interpersonnelles et sur la personnalité psychologique des personnages expliquée par leur passé. La vraisemblance de la situation est maintenue sur le fil du rasoir, à la limite du rêve et de l'hallucination, mais le rideau tombe finalement sur une explication rationnelle des événements : les visiteurs sont bien des usurpateurs et les occupants de la maison ont sombré dans la démence. Paradoxalement, c'est donc la folie des personnages qui permet d'inscrire la pièce dans le cadre réaliste! Renversant la définition classique - et morale - de la catharsis, Puig place le spectateur dans le rôle d'un expert psychiatrique, chargé de diagnostiquer les personnages.

\section{Le théâtre comme mise en abyme de la théâtralisation œdipienne}

13 Malgré le psychologisme apparent de Bajo... et la trame typiquement névrotiqueœdipienne, l'extrême ambiguïté des personnages - leur caractère "schizo » - nous incite à reconsidérer ce drame selon des critères schizo-analytiques. Puisqu'il est impossible de résumer les presque cinq-cents pages de L'Anti-EEdipe, nous nous contenterons d'en rappeler les grandes lignes, au risque de quelques simplifications schématiques... Pour Deleuze et Guattari, la théorie freudienne - trop simpliste, trop systématique quand elle ramène tout au triangle «papa-maman-moi »- a à voir avec le capitalisme ; CEdipe est une exigence ou une conséquence de la reproduction sociale :

Le névrosé reste installé dans les territorialités résiduelles ou factices de notre société, et les rabat toutes sur Ædipe comme ultime territorialité qui se reconstitue dans le cabinet de l'analyste, sur le corps plein du psychanalyste (oui, le patron, c'est le père, et le chef d'État aussi, et vous aussi, docteur...). (Deleuze \& Guattari, $1972: 42-43)$

Dans presque toutes ses œuvres, Puig fait référence à la psychanalyse et en interroge les limites, opposant notamment Lacan à Freud. Dans El beso de la mujer araña, il s'empare - en longues et érudites notes de bas de pages - de la légitimité du discours scientifique, et surtout psychanalytique, pour imposer sa propre théorie sur l'homosexualité révolutionnaire (Souquet, 2005 et 2007). Le romancier met fin à la liste, où se bousculent entre autres Freud, Fenichel, Altman et Marcuse, par une fausse référence : l'éminente doctoresse danoise Anneli Taube n'est qu'une invention, un personnage de papier, au même titre que Molina et Valentín. Face à un discours théorique "sérieux" et autoritaire, qui semblait être là pour la gloser et la cadrer, la rappeler à l'ordre, c'est 
finalement et contre toute attente, la fiction qui a ici «le dernier mot»! Daniel Balderston estime que : "[...] El cambio de sexo por el que pasa Manuel Puig al final de su tratado sobre la sexualidad (paródico, al menos parcialmente) señala que la autoridad sí puede ser cuestionada y dada vuelta, tanto en el "texto de abajo" como en el "de arriba" . » (2004:114)

Copi tourne aussi la psychanalyse freudienne traditionnelle en dérision. Dans Le Frigo, comme en écho à la malice puiguienne, l'illustre théoricien est féminisé en DoctorESSE $\mathrm{F}$ REUD et transformé en "poupée de taille humaine » (Copi, $2006: 38$ ), selon les consignes didascaliques. Tout en "manipulant » l'effigie, le comédien qui joue le rôle de L. (et de tous les autres personnages) entame un faux dialogue avec la Doctoresse, où tous les «tics» du processus thérapeutique sont parodiés et le principe même de la théorie dénoncé :

Qu'y a-t-il à l'intérieur du frigo?

Doctoresse, vous me posez des questions trop délicates!

Je n'ose pas l'ouvrir!

J'ai peur d'y trouver le cadavre de ma mère!

Elle est capable des pires plaisanteries!

Depuis sa dernière lobotomie ma mère n'est plus la même.

Heureusement le juge lui a retiré ma garde, c'est le début de ma guérison,

doctoresse, mais tout dépend de vous, doctoresse.

Vous serez bonne avec moi, doctoresse ? (Copi, $2006: 46$ )

Lorsque la psychanalyse fait du triangle œdipien familialiste un dogme, une sorte de symbole catholique universel, elle écrase la production désirante, les machines du désir : «Nous avons vu que la production désirante était la limite de la production sociale, toujours contrariée dans la formation capitaliste : le corps sans organes à la limite du socius déterritorialisé, le désert aux portes de la ville... » (Deleuze \& Guattari, 1972 : 121) Il faut donc que cette limite soit déplacée vers une territorialité factice et soumise : on rend la limite inoffensive en faisant croire qu'elle passe à l'intérieur de l'organisation molaire (la formation sociale elle-même) pour éviter la multiplicité moléculaire du désir. đEdipe "[...] déplace la limite, il l'intériorise. Plutôt un peuple de névrosés qu'un seul schizophrène réussi, non autistisé. Incomparable instrument de grégarité, đEdipe est l'ultime territorialité soumise et privée de l'homme européen" (Deleuze \& Guattari, 1972: 121). Nous sommes colonisés par la machine œdipienne-narcissique et cette territorialité intime correspond à la re-territorialisation sociale du capitalisme. C'est le sens de la dernière scène de Bajo un manto de estrellas lorsque, à la demande de ses propres parents, le médecin et l'infirmière emmènent la jeune fille - promptement diagnostiquée nymphomane - pour l'interner, on s'en doute, dans un hôpital psychiatrique - il s'agit bien de psychiatriser, d'autistiser, une schizophrénie ${ }^{12}$ trop dangereuse pour l'équilibre de la société - : «El médico despliega la camisa de fuerza y se la coloca cariñosamente mientras hablan ${ }^{13}$ » (Puig, $1983: 58$ ). C'est la «[...] causation du schizophrène artificiel, tel qu'on le voit à l'hôpital, loque autistisée produite comme entité » (Deleuze \& Guattari, 1972 : 11). Comme le rappelle Deleuze à la suite de Michel Foucault (1961), il s'agit pour la psychiatrie asilaire du XIX ${ }^{e}$ siècle, puis pour la psychanalyse freudienne, de

[...] souder la folie à un complexe parental [...] constituer un microcosme où se symbolisent «les grandes structures massives de la société bourgeoise et de ses valeurs ", Famille-Enfants, Faute-Châtiment, Folie-Désordre - faire que la désaliénation passe par le même chemin que l'aliénation, Ædipe aux deux bouts, fonder ainsi l'autorité morale du médecin comme Père et Juge, Famille et Loi [...]. (Deleuze \& Guattari, 1972 : 110) 
17 Par la voix de son personnage Pozzi, un jeune avocat péroniste de gauche, Puig montre clairement, qu'en Argentine, l'histoire de la psychanalyse et de la psychiatrie a une dimension politique ${ }^{14}$. L'action se situe en octobre 1975 (Puig, 1981: 23), en pleine répression de la « Triple A » qui, comme le rappelle Patricia Jessen, dès 1974 :

[...] assassine une soixantaine d'opposants, en majorité des avocats et des intellectuels ; début 1975, les gauchistes meurent à un rythme moyen de cinquante par semaine. [...] Le nombre de disparus augmente très rapidement. On trouve des corps flottant dans le Río de la Plata, ou brûlés, ce qui rend toute identification impossible. Des prisons filtrent les récits détaillés de tortures incessantes. (Jessen, 1990 : 91 . Je traduis.)

Or, Pozzi observe que la répression s'organise aussi au niveau des institutions psychiatriques où la dictature fait table rase d'une antipsychiatrie ${ }^{15}$ considérée subversive :

Hace dos años [en 1973] la Argentina tenía uno de los servicios psiquiátricos, gratuitos, más evolucionados del mundo. Fueron gente de la escuela inglesa de Cooper a estudiar el fenómeno, los de la Antipsiquiatría, lo más avanzado del mundo, y unos seguidores de Melanie Klein, unos franceses [...]. Cuando me venía estaban desmantelando todos los servicios psiquiátricos de los hospitales, los gratuitos. Van a dejar los manicomios nomás [...] El gobierno dice que los psiquiatras son subversivos, que tienen orientación marxista ${ }^{16}$. (Puig, $1981: 168$ )

\section{La schizophrénie marque la limite et la mort du capitalisme}

On aurait donc tort de voir les caractéristiques de Bajo un manto de estrellas - stylisation, fragilité de la vraisemblance, ambiguité et dimension archétypale (presque mythique) des personnages, psychologie, sentiments, sexualité, đEdipe... - comme une marque d'apolitisme.

Outre la convention du « quatrième mur », on ne saurait oublier un autre élément réaliste de poids : les didascalies mettent nettement l'accent sur le profil social des personnages, notamment celui des maîtres de maison qui vivent dans « una lujosa casa de campo ${ }^{17}$ » : " Son elegantes mas de una manera extremadamente sobria y opaca [...] Llama la atención especialmente que ella no se haya teñido las canas. Las ropas corresponden también al clisé que se tiene sobre la burguesía rural ${ }^{18}$. $($ Puig, 1983 : 11) Cette typologie sociale des personnages ne doit pas être considérée comme un détail, car Puig s'attache presque toujours à décrire de façon précise les modes de vie, les pratiques culturelles, les tics des milieux, avec une prédilection pour la petite bourgeoisie dont il est lui-même issu, mais portant aussi l'acuité de son regard sociologique là où on l'attend a priori le moins, quand il parle du prolétariat dans La Traición de Rita Hayworth, dans Boquitas pintadas (Keizman, 2007) ou dans Sangre de amor correspondido. Puig s'appuie ici sur une réalité objective - la prédominance de la classe moyenne et sa prégnance dans l'imaginaire identitaire de la société argentine (Souquet, 2002) - observée par nombre d'ethnologues, de sociologues ou d'historiens, à l'instar des politologues Olivier Dabène (1999) ou Alain Rouquié :

On peut même dire que la notion de classe moyenne est devenue un des mythes moteurs et comme l'axe fictif de la société nationale. Les fils d'immigrants et plus généralement des classes populaires urbaines recherchent la promotion sociale grâce aux titres universitaires et à l'exercice des professions libérales. L'engorgement des universités et la saturation de la société urbaine du littoral en 
avocats et en médecins sont entre autres une des manifestations de ce conformisme

ascensionnel. (Rouquié, $1984: 44-45)$

La focalisation didascalique sur un milieu social précis - la bourgeoisie rurale, connue pour être le ciment du conservatisme de la société argentine - montre donc que, dans Bajo..., l'analyse psychologique - voire psychiatrique - des personnages se double implicitement d'une critique sociale de la respectabilité bourgeoise et du monde des apparences. Les didascalies qui entourent les répliques du maître de maison dressent très explicitement le portrait d'un homme (socialement) arrogant et impénétrable: " (Paulatinamente recupera la flema y el aire de superioridad que le son habituales $y$, tras los cuales, oculta siempre sus verdaderos sentimientos.) ${ }^{19}{ }^{\prime}$ (Puig, $1983: 12$ )

Ce n'est pas un hasard si la première époque évoquée dans Bajo..., celle des souvenirs hallucinatoires, est délimitée par une date concrète : au-delà de la grande Dépression mondiale, 1929 marque aussi, en Argentine, la chute des gouvernements radicaux (déshonorés par les massacres d'ouvriers) et la fin des illusions démocratiques de la classe moyenne. L'extrême droite nationaliste, qui apparaît à cette époque, influencée par José Antonio Primo de Rivera et Charles Maurras, s'inscrit aussi dans la continuité du fédéralisme des caudillos du $\mathrm{XIX}^{\mathrm{e}}$ siècle en critiquant la suprématie de Buenos Aires et en idéalisant « [...] la vie rurale et les habitants des provinces, gardiens des vertus argentines et hispaniques» (Sturzenegger, 2006: 116). Les nationalistes étaient convaincus que le libéralisme conduisait au communisme, l'un et l'autre étant - selon eux - d'origine juive, ce qui «justifiait » leur antisémitisme. À partir de 1943, le gouvernement adopte une attitude complaisante à l'égard de l'Allemagne nazie, décide la dissolution des partis politiques, rend l'enseignement catholique obligatoire et pourchasse les intellectuels communistes. Les deux premières années du péronisme (1946-1948) voient une amélioration sensible du niveau de vie des classes populaires et l'instauration de lois favorables aux ouvriers (Ponce, 2001: 76-77), mais la deuxième date de Bajo un manto de estrellas, 1948, celle du présent de l'action dramatique, est entachée par le rapprochement entre Perón et Franco et, donc, par la confirmation des sympathies fascistes du leader populiste $^{20}$. Comme le note Graciela Goldchluk, le climat général de la pièce - pourtant dénuée de références historiques et politiques explicites - est marqué par « un ambiente en que el mundo parece desintegrarse $e^{21} »(1983: 9-10)$.

21 L'acmé dramaturgique de Bajo... se situe au moment de la scène de défloration de la jeune fille par le mystérieux visiteur. Comme le souligne M. Ezquerro, «[...] le fantasme d'inceste entre père et fille, de défloration de la fille par le père est à la fois réalisé et nié, grâce au dédoublement du rôle du père en deux personnages " (1983: 50). De plus, les paroles du père adoptif au moment où il surprend la scène - «[...] somos una alucinación ${ }^{22}$ »(Puig, 1983: 34) -, qui libèrent la jeune fille des contingences sociales et morales et l'encouragent à continuer de faire l'amour devant ses parents, peuvent être interprétées de deux façons différentes : d'un côté, ce discours déculpabilisant semble positif, mais il correspond aussi à un déni du réel, ce que confirme nettement l'une des dernières répliques du personnage : «[...] no podría haber sido tan triste nuestra suerte. Ella no perdió la razón, y no se la llevaron a un manicomio. Está aquí, jugando con nosotros como cuando niña ${ }^{23}$ " (Puig, 1983 : 62). La mise en évidence des tabous de la morale sexuelle s'articule ici avec une critique politique de la société. Par le biais de ces personnages autistiques, c'est - comme dans ses deux premiers romans - la bourgeoisie provinciale et, finalement, toute la société argentine dont Puig vise le puritanisme hypocrite, pratiqué dans la classe moyenne par imitation des élites ${ }^{24}$. Malgré l'importance des apparences, des conventions, 
de la bienséance, c'est cette société très «décente » et "convenable », obsédée par la notion de respectabilité, qui va pourtant permettre passivement - ou favoriser activement - la montée de l'extrême droite et l'installation des dictatures. Pour Puig, la société argentine est, depuis 1929, dans le déni constant de sa fascination pour le fascisme. Alors que Freud s'arrête aux névroses sexuelles individuelles, son disciple hétérodoxe Wilhelm Reich analyse, à l'échelle de la société entière, le rôle de la sexualité dans l'asservissement des peuples. Comme Deleuze citant La psychologie de masse $d u$ fascisme de Reich, Puig ${ }^{25}$ refuse de considérer la dictature comme une fatalité et de déresponsabiliser les individus :

Jamais Reich n'est plus grand penseur que lorsqu'il refuse d'invoquer une méconnaissance ou une illusion des masses pour expliquer le fascisme, et réclame une explication par le désir, en termes de désir : non, les masses n'ont pas été trompées, elles ont désiré le fascisme à tel moment, en telles circonstances, et c'est cela qu'il faut expliquer, cette perversion du désir grégaire. (Deleuze \& Guattari, 1972 : 37)

Alors qu'il expose déjà dans ses romans les contradictions de sa passion pour un cinéma d'évasion, qui endoctrine le public mais joue malgré tout une fonction cathartique, Puig trouve avec l'écriture dramatique un nouveau moyen d'étoffer sa réflexion sur les rapports entre simulacre et vérité. Dans un décor spécifiquement " post-art-nouveau et pré-art-déco ", qui rappelle les écrins des comédies de Cukor ou de Lubitsch (Berthomé, 1991: 87-95), Bajo un manto de estrellas / Sous un manteau d'étoiles (le titre évoque évidemment les étoiles d'Hollywood) condense le mensonge, le vol, la luxure, l'adultère, l'inceste, le suicide, le meurtre... et transgresse tous les interdits que le code de censure Hays posait dans le cinéma hollywoodien, notamment entre 1929 et 1948 (les deux dates clés de l'intrigue), grande période de l'embourgeoisement de l'imaginaire cinématographique et de cette stylisation du réel que Puig préconise dans son introduction didascalique. C'est probablement, pour lui, le moyen de réaliser la synthèse entre son idéal esthétique et sa quête d'une éthique "schizo » qui brise le carcan d'une morale bourgeoise dont il est à la fois le fruit et la victime. Il est d'autant plus jouissif pour un auteur de faire éclater les conventions sociales sur scène que - selon Étienne Fuzellier (1964) - le théâtre est un genre particulièrement "conventionnel et symbolique ». Or, dans cette pièce qui jongle avec les protocoles du mélodrame rétro et du théâtre réaliste bourgeois, les conventions sociales se dérobent et s'abolissent en même temps que les conventions théâtrales. M. Ezquerro montre que la seule façon de représenter la scène d'inceste est de la "déréaliser » en la désignant explicitement comme un fantasme, ce que font les personnages, dans un mouvement métathéatral où ils glosent inlassablement les thèmes du masque et de l'illusion: "Le théâtre, mode privilégié de la représentation, pourrait-il ne pas représenter son propre fonctionnement » (Ezquerro, 1983: 58) ? En prolongeant ce constat, nous voyons que si Bajo... est une mise en scène de la théâtralité et d'Æđdipe, elle est aussi la démonstration qu'CEdipe est une mise en scène élaborée à des fins politiques !

Deleuze pense que le "freudisme", c'est-à-dire la tendance la plus conservatrice et institutionnelle de la psychanalyse, est là pour exercer un contrôle des consciences et éviter la multiplicité moléculaire du désir, énergie libre capable d'alimenter la machine révolutionnaire. Engageant «[...] le désir dans les impasses œdipiennes du couple et de la famille au service des machines répressives [...] » (Deleuze \& Guattari, 1972: 349), CEdipe nous colonise et nous enferme dans la territorialité sociale molaire. Pour Deleuze, le narcissisme œdipien est une mise en scène, un cirque, un théâtre au service du 
capitalisme : « Pourquoi avoir installé des formes expressives, et tout un théatre là où il $\mathrm{y}$ avait des champs, des ateliers, des usines, des unités de production? Le psychanalyste plante son cirque dans l'inconscient stupéfait, tout un Barnum aux champs et dans l'usine. » (Deleuze \& Guattari, 1972 : 354) Deleuze fait alors appel au Hamlet d'Henry Miller - la tragédie d'Hamlet est un équivalent du mythe d'ÆEdipe - pour montrer qu'il n'y a pas de vie possible dans la camelote idéologique du mythe. La schizo-analyse sert à nettoyer - à « cureter " - l'inconscient de ces croyances et illusions que sont le mythe et la tragédie. La schizo-analyse est un processus schizophrénique de déterritorialisation qui doit produire «[...] le fonctionnement des machines désirantes contre la tragédie, contre "le funeste drame de la personnalité", contre "la confusion inévitable du masque et de l'acteur" » (Deleuze \& Guattari, 1972: 356). C'est ce «curetage » que Puig opère sur les chefs-d'œuvre populaires dont il se nourrit, en les débarrassant de leur idéologie (parfois) réactionnaire pour n'en garder que la quintessence artistique. La pièce de Puig interroge le théâtre comme lieu même du simulacre et questionne aussi la société comme autre simulacre, tout aussi artificieux mais dans le déni complet de sa fausseté.

Le théâtre réaliste implique un choix éthique: celui du respect des conventions. Avec Bajo..., Puig inscrit pleinement dans l'écriture dramatique la question qu'il pose dans toute son œuvre : faut-il respecter les conventions sociales ou prendre le risque de choisir son vrai désir? L'identité fluctuante de ces personnages schizos, leur folie réelle ou supposée, se redouble dans l'hésitation constante entre les conventions réalistes et le caractère absurde de la pièce, ligne d'indécision relayée par le rappel et la transgression simultanée des principes traditionnels de la dramaturgie (catharsis, « quatrième mur »). Le caractère de la pièce semble toujours indécidable: convention ou transgression? Pièce psychologique, métaphysique, ou politique? Ici, la figure du fou (la fille) libère une écriture du retournement: les vrais fous sont les parents, prisonniers comme toute la société argentine des conventions.

\section{Des acteurs et des infirmières pour un « théâtre maïeutique »}

Prolongeant la réflexion engagée, par Bajo un manto de estrellas, sur les liens paradoxaux entre le théâtre réaliste, la distanciation, la catharsis et la schizophrénie, Puig écrit $E l$ Misterio del ramo de rosas (1987), une pièce en deux actes, d'abord publiée en italien sous le titre Misterio del mazzo di rose puis dix ans plus tard, en 1997, en espagnol. L'œuvre a été interprétée par des comédiennes aussi prestigieuses qu'Anne Bancroft et Jane Alexander (Los Angeles, 1989) ou Dominique Sanda et Cristina Banegas (Multiteatro, Buenos Aires, 2006). Duo de femmes, donc, comme souvent chez Puig: PACIENTE (Patiente) et ENFERMERA (Infirmière). Une riche bourgeoise arrogante (comme il se doit), acariâtre (à souhait) et dépressive depuis la mort de son petit-fils, se fait interner volontairement dans une clinique privée de luxe où elle reçoit les soins d'une infirmière, d'autant plus dévouée qu'elle est au service exclusif et à la solde de la malade. À 47 ans, la soignante, issue d'un milieu modeste, vit dans l'amertume de n'avoir pu étudier la médecine et d'avoir laissé échapper son seul amour, cédant aux préjugés de ses parents. Elle considère le monde comme un cloaque mais décide, pour garder sa place, de mettre en œuvre une étrange thérapie personnelle, faite de dialogue et de négociation. Peu à peu, se tisse une relation trouble où l'on ne sait plus qui mène le jeu. La patiente autoritaire, une avocate qui a renoncé à exercer pour se consacrer à un mari qui la trompait sans vergogne, tente de 
sortir son infirmière de la soumission sociale, sans cesser pourtant de la houspiller et de la rabrouer: "Pero dígame un poco, ¿no le da vergüenza dejarse tratar así?, ¿o lo que le pago me da derecho al maltrato? „jconteste!! ${ }^{26} »($ Puig, 1997 : 99)

L'inconscient s'en mêle quand les deux femmes sont, tour à tour, prises par des hallucinations introspectives où elles revivent des moments clefs de leur vie. C'est ici que le génie dramatique de Puig s'exprime: les personnages secondaires et hallucinatoires, des défuntes qui surgissent de la mémoire des deux femmes, sont incarnés par les mêmes actrices (la sœur de la patiente est jouée par l'infirmière et la mère de l'infirmière par la patiente). Ce procédé, qui rappelle évidemment celui de Bajo..., souligné par des changements de lumières, met en exergue la dualité schizophrénique de personnages sur lesquels plane constamment la menace d'une instrumentalisation, d'une trahison, et dont on ne sait jamais s'ils sont manipulateurs ou manipulés, victimes ou bourreaux: « $P$ ACIENTE. - (Vuelve la iluminación realista, la Paciente recobra su personalidad habitual, se encuentra en la misma posición del momento en que se produjo el corte fuera de la realidad.) ¿no me entiende?, ¿por qué se queda mirándome así? ${ }^{27}$ »(Puig, 1997 : 99) La patiente sort peu à peu de son abattement et redevient « impatiente » / " Impaciente » (Puig, 1997 : 114) de vivre, grâce à la "patience » de l'infirmière, qui lui propose d'échanger leurs rôles, comme dans une clinique expérimentale dont elle a entendu parler : "Recuerde que ahí no hay tal categoría, de sanos y enfermos. Todos son enfermos ${ }^{28} »$ (Puig, 1997 : 127). Patiente et infirmière seront finalement guéries - sauvées - par la parole. Éloge de la maïeutique qui accouche les esprits d'un savoir caché en eux, cette superbe pièce de Puig - où le personnage «mineur » de l'infirmière devient «médecin du monde »- nous offre une réflexion très achevée sur la fonction cathartique et curative du théatre, faisant écho à l'idéal d'Artaud: "Rejoindre les passions par leurs forces, au lieu de les considérer comme des abstractions pures, confère à l'acteur une maitrise qui l'égale à un vrai guérisseur. » (1964 : 202-203)

L'infirmière - ce n'est pas un hasard - est un personnage dramatique récurrent puisqu'elle apparaît aussi dans Eva Peron (1969) et Une visite inopportune (1988), deux pièces de Copi qui, d'une certaine manière, se répondent. Toutes ces infirmières, uniquement désignées par leur fonction, sont des personnages anonymes qui se vivent comme tels : «INFIRMIÈRE. - [...] Je serai toujours une personne anonyme, mais je vous promets que mes enfants, je les ferai tous acteurs, comme vous, pour qu'ils deviennent célèbres. CYrille. - Si vous cherchez la célébrité, faites-les plutôt médecins. » (Copi, 1999b: 64) Modestes, humbles et dévouées, si "effacées »-grâce à une incroyable économie de parole et de jeu - qu'elles en deviennent presque transparentes, au point d'être sacrifiée, chez Copi, pour jouer la doublure mortelle d'Eva Perón. Leur insignifiance, qui les place apparemment à l'opposé de l'acteur («l'acteur est un "monstre" ", nous dit Deleuze [1985: 97]), est un défi à la théâtralité des personnages. Puig et Copi renouent avec le thème baroque du " grand théâtre du monde » où le pouvoir appartient aux hypocrites, c'est-à-dire, étymologiquement, aux acteurs. Mais cette puissance est aussi complètement réversible puisque le jeu mène à la découverte de la vérité: l'absence de sens et, partant, l'absurde. Face à l'absurdité du monde, l'acteur devient impuissant, incapable d'agir, négation de lui-même :

HUbert. - Vous rêvez, Cyrille. C'est ce soir que vous allez jouer « Hamlet » pour la première fois. Apprêtez-vous à entrer en scène.

CYRILLE. - Entrer en scène ? Mais je suis en train d'en sortir !

HUBERT. - C'est la vie de théâtre, quand c'est fini c'est pour recommencer. [...]

CYRILLE. - Je n'ai pas joué «Hamlet » depuis un siècle! Je ne me souviens même 
plus du texte...

HUBERT. - Jouez n'importe quel personnage, ils se valent tous. (Copi, 1999b : 68) dont elles n'ont pas le pouvoir - rappelons-nous que le freudisme a confirmé «[...] l'autorité morale du médecin comme Père et Juge, Famille et Loi [...]» (Deleuze \& Guattari, 1972: 110) -, les infirmières incarnent la minorité : vouées aux tâches les plus ingrates, ces « bonnes » de la médecine (l'infirmière d'Une visite... se voit d'ailleurs obligée de rappeler au professeur qu'elle n'est pas sa bonne) représentent le personnage adjuvant par excellence, ancillaires mais indispensables, véritables Carons des hôpitaux, elles réparent tous les maux, jusqu'à la mort elle-même puisque l'infirmière d'Eva meurt à sa place et que celle de Cyrille (le double de Copi) s'exclame, avant que le rideau ne tombe : « Zut! J’avais oublié que vous étiez mort ! (Copi, 1999b : 73) Réplique sublime, revanche suprême des opprimés : dans cette ultime pièce de Copi, c'est donc l'infirmière qui aura non seulement le dernier mot - réparateur - mais la réplique unique de la dernière scène !

\section{Les quatre jumelles de Copi ou l'agonie perpétuelle}

Cette pièce fut créée le 29 octobre 1973, au Palace, dans le cadre du Festival d'automne. La mise en scène était de Jorge Lavelli et les quatre rôles étaient tenus par Daisy Amias, Myriam Mézières, Liliane Rovère et la géniale Anna Prucnal.

Le titre, en lui-même, malgré la limpidité de sa signification, joue déjà sur le paradoxe et l'absurde : le cardinal quatre semble contredire la dualité gémellaire alors, qu'en fait, elle la double dans un jeu de miroirs qui fait délirer le baroque. Les personnages ont-ils des visions ? À moins que ce ne soit le spectateur !...

31 L'action se déroule dans un lieu unique, chez les jumelles Maria et Leïla Smith (allusion possible aux Smith de La Cantatrice chauve chez un auteur qui joue constamment avec l'intertextualité). Les jumelles vivent en Alaska. Un Alaska fantastique, bien sûr, nullement référentiel, rappelant plutôt l'enfer de Huis clos. Là, les sœurs Smith rencontrent les sœurs Goldwashing : Joséphine et Fougère, jumelles elles aussi. Formant un atroce quadrille, les deux «paires de jumelles » sont unies par une même cupidité et les signes de l'argent (dollars, or, émeraudes, diamants) constituent l'un des leitmotive de la pièce. Les riches sœurs Smith sont dans la veine provocatrice et subversive des personnages du Charme discret de la bourgeoisie de Buñuel. Leur statut dominant tient au seul capital économique, acquis par la voie du crime: «Regarde-la comme elle joue les saintes-nitouches quand elle vient de tuer sa sœur! C'est des vraies salopes, ces femmes!» (Copi, 1999a : 19) Les bourgeoises sont, en fait, des braqueuses de banques et de bijouteries, consommatrices et trafiquantes de drogue... deux postulats sont dès lors envisageables : soit il s'agit de fausses bourgeoises ou alors... la respectabilité bourgeoise n'est jamais qu'une façade servant à dissimuler la violence de la domination exercée par cette classe. On retrouve - détournée - l'opposition entre les héros victimes et les personnages persécuteurs, propre au théâtre de Strindberg et reprise par Adamov puis par la dramaturge argentine Griselda Gambaro, notamment dans la pièce Los siameses (1965) (Urdician, 2006, 2009), ou encore chez l'Uruguayen Ricardo Prieto, dans Los disfraces (1968) où le domestique et la jeune fille de la maison échangent leurs rôles et se livrent à un cruel jeu sado-masochiste avant de retrouver leurs statuts respectifs... Les sœurs Goldwashing sont évidemment héritières des Bonnes (1947) de Genet, pièce dont la 
thématique du double et l'esthétique de la « cérémonie » fascinaient Copi et qu'il avait lui-même interprétée. Comme le souligne Frédérique Mengard, «[...] dans Les Bonnes de Genet, le jeu supplante la réalité, le miroir déformant du monde se fait plus vrai que le monde lui-même » (2003 : 100). Claire, l'une des deux bonnes qui s'amusent à porter les robes de leur maitresse et à l'imiter, accomplit son destin « schizo » en buvant le poison qu'elles avaient destiné à leur patronne et meurt donc à sa place, détruisant sa propre existence dans le fantasme - thème que Copi reprendra dans Eva Peron où l'infirmière meurt à la place d'Eva. On peut aussi supposer une influence de la pièce du Cubain de Paris Eduardo Manet, Les Nonnes (1968), où trois truands se déguisent en religieuses pour enlever une femme qu'ils dévaliseront, tueront, et dont ils transformeront le corps en image pieuse.

Mais la société vue par Copi ne saurait être manichéenne et les prolétariennes Goldwashing, entièrement déterminées par leur patronyme (littéralement: "lavage d'or ») ne sont pas plus ingénues et "innocentes» que les Smith, les vraies-fausses bourgeoises :

Je vais vous raconter un peu notre histoire. Nous sommes très pauvres. Nous sommes nées comme ça et il n'y a rien à faire, nous ne gardons aucune place parce que ma sœur se drogue, et en plus on nous chasse de partout parce qu'on s'attire des emmerdements, qu'on le veuille ou non, à cause de mon mauvais caractère. C'est là que ma sœur a eu l'idée de venir chercher de l'or en Alaska, et nous voilà. Mais nous n'avons rien trouvé [...] Mais ce qu'on souhaite, au fond, c'est de trouver une bonne place stable auprès d'une personne gentille comme vous. Ma sœur fait très bien la cuisine et moi je suis très forte pour la lessive. (Copi, 1999a : 17-18)

Évidemment, les patronnes ne sont ni dignes ni convenables et surtout pas « gentilles » et les bonniches chercheuses d'or pas si serviles... Nait alors un combat sans merci, une lutte à mort sans fin pour récupérer le magot des Smith. Pourtant, ce qui semble être une opposition de classes, n'est en fait qu'une lutte fratricide - «sœuricide» ou même "gémellicide», aurait probablement «néologisé » Copi- (les paires sont interchangeables car les quatre personnages se ressemblent et se valent), allusion probable aux guerres civiles "décimononiques» et autres guérillas - parfois fratricides - qui déchirèrent le Río de la Plata et peut-être, plus spécifiquement, au massacre argentino-argentin d'Ezeiza - opposant péronistes de gauche et péronistes de droite : bonnet blanc et blanc bonnet comme Smith et Goldwashing ?...-, carnage quasi mafieux qui vient d'avoir lieu quatre mois avant la création de la pièce ${ }^{29}$.

Très vite, l'action bascule dans l'absurde et le fantastique - un fantastique souvent ironique, chez Copi comme chez Arenas ou F. Vallejo, pour dire l'impuissance de la raison et son insuffisance - quand l'une des jumelles précédemment occise ressuscite, initiant le cycle infini des agonies et des morts provisoires et constamment réparées de chaque personnage. Par le biais de Leïla, la pièce est implicitement placée sous le signe de Lilith - sa mère en mythologie - l'âme noire du monde et de la mort (Durand, 1984:114). Ces jumelles sont - ou semblent - immortelles et se voient donc condamnées à s'entretuer indéfiniment. Le spectateur peut d'ailleurs se demander si l'action est censée être "réelle» ou si elle est le fruit d'une hallucination, d'une vision, car les corps des personnages sont confits de sniffs et d'injections de toxiques hallucinogènes (piqûres de talc ou de camphre, morphine, amphétamines, cocaïne, héroïne) :

FOUGÈRE. - Je suis fichue ! J'ai plein de balles incrustées dans la rate, les reins et les

poumons ! Cette fois-ci je crève pour de bon, ma petite!

JosÉPHINE. - Ne crève pas! Je vais te faire une piqûre de camphre ! [...] 
FoUGÈrE. - [...] Tire-toi vivante avant qu'elles ne ressuscitent ! [...]

JosÉPHINE. - Je vais te faire extirper toutes les balles! On a un million de dollars !

Un million de dollars verts comme des émeraudes! Un million de dollars ! C'est pas

le moment de mourir ! Lève-toi ! Lève-toi ! Lève-toi! (Copi, 1999a : 43-44)

Lève-toi et «vole» (ici dans les deux sens du terme)! Et le miracle se produit! Les hallucinogènes les font délirer et elles s'envolent sur une "ligne de sorcière ", elles ont des visions, elles voient des dollars, de l'or, des émeraudes qui leur permettent de s'inscrire dans un devenir immortel. Comme nous le rappelait Bataille, dans La littérature et le mal, la pauvreté rapproche de la mort: c'est pour oublier qu'il est mortel que l'homme cherche la richesse. Il n'y a pas de littérature sans fabulation, souligne Deleuze (1993:13), mais «[...] la fonction fabulatrice ne consiste pas à imaginer ni à projeter un moi. Elle atteint plutôt à ces visions, elle s'élève jusqu'à ces devenirs ou puissances ».

\section{Éliminer les « maîtres »}

Les jumelles, saint(es) Lazare parodiques mais véritables " ordures ", " salopes ", comme elles se le répètent sans cesse, se relèvent toujours, increvables comme les mythes haineux - opposant civilisation et barbarie - qui fomentèrent le racisme social et ethnique de la République argentine et la plongèrent, en 1976, trois ans après Ezeiza, dans la plus sanguinaire de ses interminables dictatures. L'excès de haine et de violence interroge ici la fonction cathartique du théatre et son rapport au réel. Comme Puig, avec "l'air de ne pas y toucher ", Copi se livre à un véritable "curetage» des conventions théâtrales, sociales et politiques, et à une mise à plat des grands mythes civilisationnels.

Alors, pièce politique ou pas? Oui... mais certainement pas comme on l'entend généralement... L'histoire ancestrale des maîtres et valets (qu'Octave Mirbeau raconte si bien, puis Buñuel, et pas seulement dans Le Journal d'une femme de chambre) fait ici volteface: Copi dépasse le simple renversement carnavalesque maîtres-valets, il élimine les "maîtres », les bourreaux deviennent bourrelles puis, à leur tour, victimes. Ces quatre personnages n'ont pas vraiment de caractères formels, ils-elles sont plutôt dans ce que Deleuze appelle une "zone de voisinage»: entre les milieux, entre les classes. Les patronnes potentielles ne maitrisent pas leur rôle (elles ne savent pas se faire respecter), pas plus que les pauvres ne maitrisent le leur: elles cherchent de l'or mais n'ont pas d'outils, elles prétendent vouloir être « bonnes à tout faire » mais elles ne sont « bonnes à rien ", quand elles disent qu'elles ne gardent aucune place, il faut entendre qu'elles ne restent pas en place, (socialement) pas à "leur " place, contrairement à ce qu'elles affirment, elles ne sont ni dévouées ni serviles, elles ne se soumettent pas :

La ligne de variation, ne passe pas entre les maîtres et les esclaves, ni entre les riches et les pauvres. Car, des uns aux autres, se tisse tout un régime de relations et d'oppositions qui font du maître un esclave riche, de l'esclave un maittre pauvre, au sein d'un même système majoritaire. (Deleuze, 1979 : 126)

Et le système majoritaire, ici, c'est la violence générée par la quête effrénée de l'argent: c'est la cupidité et le pouvoir mortifère de l'argent. Copi anticipe donc, implicitement, le constat que fera Sloterdijk, quelques années plus tard. Souvenons-nous de ce que disait le philosophe de la postmodernité à propos de la métaphore du " palais de cristal » :

Du capitalisme, il a fallu cependant attendre nos jours pour dire qu'il a toujours représenté plus qu'un « rapport de production »; depuis toujours, sa prégnance a largement dépassé ce que la figure intellectuelle du «marché mondial » pouvait désigner. Il implique le projet consistant à transposer la totalité de la vie du travail, 
des désirs et de l'expression artistique des êtres, dans l'immanence du pouvoir

d'achat. (Sloterdijk, $2006: 253$ ) chez Puig, dans des personnages tels que Nelly: «Mamá me dijo que metiéndome donde no me correspondía iba a ser para lío nada más. Dicho y hecho ${ }^{30}$.» (Puig, $\left.1985: 25\right)$ Le roman Boquitas pintadas (1969) repose notamment sur les croisements entre les classes sociales: histoires multiples d'ascensions ou de tentatives d'ascension et de déchéances, vases communicants qui relativisent ou annulent les rapports de forces entre dominants et dominés.

\section{Amputer l'Histoire}

Mais revenons à Copi. Quelle est la trame des quatre jumelles, quelle est l'histoire ? C'est la cupidité comme moteur de l'action - nous l'avons vu-, mais il n'y a aucun repère historique : la cupidité « capitaliste» est donc rabattue vers une dimension ahistorique. Comme le remarquait déjà César Aira (2003:112) - dans une perspective implicitement deleuzienne -, dans cette pièce tout est centre ${ }^{31}$ : dès la première réplique, à quelques secondes du début, la pièce est déjà à son maximum d'intensité et s'y maintient jusqu'à la fin - et virtuellement jusqu'à l'infini - puisque la catastrophe suprême, le climax tragique que constitue la mort du héros survient sans cesse puis s'annule...

Aira distingue aussi deux types d'histoire dans L'Homosexuel ou la difficulté de s'exprimer (1971) : l'aventure et l'histoire proprement dite. L'aventure, c'est ce qui se passe sur scène (la préparation d'une fuite vers la Chine) tandis que l'histoire biographique d'Irina (son enfance, son changement de sexe, ses voyages, ses amants) peine à s'exprimer. Prolongeons les remarques d'Aira. Les jumelles..., comme presque toujours chez Copi, comme L'Homosexuel..., est une pièce du temps miniaturisé, "minoré », une pièce de l'instant qui se développe par le milieu : l'histoire (ce qu'Aira appelle l'aventure) se voit réduite à la portion congrue et semble flotter dans la mer du temps, elle ne parvient pas à s'inscrire dans la « grande » Histoire : « [...] il faut amputer l'histoire, parce que L'Histoire c'est le marqueur temporel du Pouvoir", nous dit Deleuze (1979: 103). Il n'y a donc presque pas de trame (action, aventure) ni d'histoire biographique des personnages parce que surinvestir ces histoires-là ce serait comme leur donner une dimension historique, vouloir les raccrocher à l'Histoire (leur donner un passé et un avenir plus qu'un devenir) et, donc, les lier au Pouvoir et à son discours. La frontière passe entre l'Histoire et l'antihistoricisme, chez Copi comme chez Puig, la pièce se situe dans cette anfractuosité où le théâtre parvient à raconter l'histoire de ceux dont l'Histoire ne tient pas compte, mais sans tomber dans le piège de l'historicisme et du didactisme qui ramènent l'œuvre d'art dans le champ du molaire. Lorsque Fougère Goldwashing dit : «Je vais vous raconter un peu notre histoire. Nous sommes très pauvres », le « un peu » est essentiel. C'est d'ailleurs aussi le sens de la réplique de l'infirmière, l'une des deux protagonistes du Misterio del ramo de rosas de Puig: « Y a lo mejor las que están olvidadas sí que servirían de algo... ${ }^{32}$ » (1997: 92) Cette perspective rejoint partiellement celle de Benjamin : il faut raconter l'histoire des anonymes, pas celle des grands héros de l'Histoire.

Le théâtre de Puig - mise en abyme de la théâtralisation œdipienne - et celui de Copi - «maniériste ", « inopportun» (Une visite inopportune) et sans complaisance - sont parfaitement opératoires. Selon une perspective souvent métathéâtrale, «mineure » et 
révolutionnaire mais universelle (oscillant entre métaphysique et politique), et malgré une distance apparente, ils interrogent profondément l'identité argentine.

\section{BIBLIOGRAPHIE}

AIRA César (2003), Copi, Rosario, Argentine : Beatriz Viterbo Editora.

AlloUCH Jean (1983), « Exorbitantes sœurs Papin », Littoral, 9, 127-146.

ARTAUD Antonin (1964), Le Théâtre et son double, présentation biographique de Paule Thévenin, Paris : Gallimard.

Avellaneda Andrés (1993), « Argentina militar: los discursos del silencio », K. Kohut et A. Pagni (coord.), Literatura argentina hoy: de la dictadura a la democracia (p. 13-15), Francfort-sur-le-Main : Vervuert Verlag.

BARÈGE Thomas (2013), « Une visite inopportune de Copi : un testament français ? Lecture intertextuelle », F. Morcillo et C. Pélage (dir.), Littératures en mutation. Écrire dans une autre langue (p. 159-172), Orléans : Paradigme.

BERTHOMÉ Jean-Pierre (1991), « Le décor : l’hégémonie de Cedric Gibbons », A. Masson (dir.), Hollywood 1927-1941, La propagande par les rêves ou le triomphe du modèle américain (p. 87-95), Paris : Autrement.

BALDERSTON Daniel (2004), « Sexualidad y revolución: en torno a las notas de El beso de la mujer araña ", El deseo, enorme cicatriz luminosa, Ensayos sobre homosexualidades latinoamericanas, Rosario, Argentine : Beatriz Viterbo Editora.

BONARDI Laurent (2004), « L'image de l'Argentine péroniste dans la presse espagnole (1946-1955) », El Argonauta Español, 1, <http://argonauta.imageson.org/document47.html>.

CAPEllán Jesús Antonio (1995), « El teatro de Manuel Puig », Teatro, Revista de Estudios Teatrales, 6-7 , 163-176.

CELIK Olivier (2006), « Théâtre à jouer, théâtre pour jouer », entretien avec Marcial Di Fonzo Bo et Elise Vigier, Copi, Loretta Strong / Le Frigo, Paris, L'Avant-scène théâtre, 1204, 61-64.

CoPI (1971), L'Homosexuel ou La difficulté de s'exprimer, Paris : Christian Bourgois.

COPI (1999a), Les Quatre jumelles (1973), suivi de La tour de la Défense (1978), Théâtre I, postface de Colette Godard, « Copi le voyageur », Paris : Christian Bourgois.

Copi (1999b), Une visite inopportune (1988), avec des textes de Cavana, Michel Cournot, Guy Hocquenghem, Jorge Lavelli, Jacques Sternberg, Paris : Christian Bourgois.

Copi (2003), Eva Peron (1969), Paris : Christian Bourgois.

COPI (2006), Le Frigo / Loretta Strong, Paris, L'Avant-scène théâtre, 1204.

DABÈNE Olivier (1999), L'Amérique latine au XXe siècle, Paris : Armand Colin. 
DEJONG Nadine (1999), «Le Baiser de la femme-araignée : écriture dramatique ? », Rencontre autour de la pièce Le baiser de la femme araignée de M. Puig, Puente, Bulletin de la S.B.P.E., spécial Manuel Puig, 98, 8-17.

DelaVAud Gilles (2005), L'art de la télévision. Histoire et esthétique de la dramatique télévisée, Bruxelles : De Boeck Université.

Deleuze Gilles \& Guattari Félix (1972), L’Anti-CEdipe. Capitalisme et Schizophrénie, Paris : Minuit. Deleuze Gilles (1979), « Un manifeste de moins », C. Bene et G. Deleuze, Superpositions, Richard III (traduit de l'italien par J.-P. Manganaro et D. Dubroca), suivi de « Un manifeste de moins », Paris : Minuit.

DeleuZe Gilles (1985), Image-temps. Cinéma 2, Paris : Minuit.

Deleuze Gilles (1993), « La littérature et la vie », Critique et clinique, Paris : Minuit.

Deleuze Gilles (2003), Deux régimes de fous, Paris : Minuit.

DUBATTI Jorge (1990), «El teatro del absurdo en Latinoamérica », Espacio de Crítica e Investigación Teatral, 8, 115-123.

DUBATTI Jorge (1999), El teatro laberinto. Ensayos sobre teatro argentino, Buenos Aires : Atuel.

DuBATTI Jorge (2002), quatrième de couverture, Copi, Cachafaz / La sombra de Wenceslao, Buenos Aires : Adriana Hidalgo.

DURAND Gilbert (1984), Les structures anthropologiques de l'imaginaire, Paris : Dunod.

EZQUERRo Milagros (1983), «Le fonctionnement sémiologique des personnages dans Bajo un manto de estrellas de Manuel Puig ", Caravelle, Cahiers du monde hispanique et luso-brésilien, 40, Le Théâtre en Amérique latine, 47-58.

Foucault Michel (1961), Histoire de la folie, Paris : Plon.

FUZELLIER Étienne (1964), Cinéma et littérature, Paris : CERF.

GoLDCHLUK Graciela (1983), « Prólogo », M. Puig, Bajo un manto de estrellas / El beso de la mujer araña (adaptación teatral) (p. 9-10), Barcelone : Seix Barral.

Guzzo Cristina (2004), « Luisa Capetillo y Salvadora Medina Onrubia de Botana: dos íconos anarquistas. Una comparación », Alpha, 20, 165-180, <www.scielo.cl/scielo.php? script=sci_arttext\&pid=S0718-22012004000200011\&lng=es\&nrm=iso>.

JesSEn Patricia B. (1990), La realidad en la novelística de Manuel Puig, Madrid : Pliegos.

JOECKER Jean-Pierre (1981), « Entretien avec Copi », Masques, Revue des homosexualités, 11, 33-38.

KeIzMAn Betina (2007), «Les autres traces (une relecture de Boquitas pintadas de Manuel Puig, Cabecita negra de Rozenmacher et El juicio de Dios de Di Benedetto) », Le texte et ses liens II, Université Paris-Sorbonne, Les Ateliers du séminaire Amérique latine, sous la direction de Milagros Ezquerro, réalisation Julien Roger, <www.crimic.paris4.sorbonne.fr/actes/tl2/texteliens2.htm>.

LACAN Jacques (1972), « Motifs du crime paranoïaque : le double crime des sœurs Papin », Obliques, $2,100-103$

LACRoIX Jean (2006), « La signification de la folie selon Michel Foucault », PhiloSophie, Académie de Grenoble, <www.ac-grenoble.fr/PhiloSophie/articles.php?lng=fr\&pg=860>. 
LATEANA Lia Beatriz (2005), « Une ébauche d'approche socioterminologique. La notion de schizophrénie en langue espagnole », Revue de la S.A.P.F.E.S.U. (Sociedad Argentina de Profesores de Francés de la Enseñanza Superior y Universitaria), 28, 40-46.

LINENBERG-FRESSARD Raquel (1987), Exil et langage dans le roman argentin contemporain : Copi, Puig, Saer, avec un entretien réalisé avec Copi (thèse de troisième cycle dirigée par A. Bensoussan), Université Rennes 2 - Haute Bretagne.

MENGARD Frédérique (2003), Les comédies de Friedrich Dürrenmatt ou le théâtre considéré comme institution morale (thèse de doctorat dirigée par J.-L. Bandet), Université Rennes 2 - Haute Bretagne.

Míguez Eduardo J. (1999), « Familias de clase media: la formación de un modelo », F. Devoto et M. Madero (dir.), Historia de la vida privada en la Argentina, t. II : La Argentina plural: 1870-1930 (p. 21-45), Buenos Aires : Taurus.

Pauls Alan (1986), Manuel Puig: La Traición de Rita Hayworth, Buenos Aires : Librería Hachette S.A.

Ponce Néstor (2001), L'Argentine, crise et utopies, Paris : Éditions du Temps.

PUIG Manuel (1981), Pubis angelical, Barcelone : Seix Barral.

PUIG Manuel (1981), Pubis angélical (trad. d'Albert Bensoussan), Paris : NRF, Gallimard.

PUIG Manuel (1983), Bajo un manto de estrellas / El beso de la mujer araña (adaptación teatral), Barcelone : Seix Barral.

PUIG Manuel (1985), Boquitas pintadas, Barcelone : Seix Barral.

PuIG Manuel (1997), El misterio del ramo de rosas, précédé de Bajo un manto de estrellas, édition de G. Goldchluk et J. Romero, Buenos Aires : Beatriz Viterbo Editora.

RoUQUIÉ Alain (1984), L'Argentine, Paris : PUF.

SALESSI Jorge (2000), Médicos maleantes y maricas, Higiene, criminología y homosexualidad en la construcción de la nación Argentina. (Buenos Aires : 1871-1914), Estudios culturales, Rosario, Argentine : Beatriz Viterbo Editora.

SLOTERDIJK Peter (2006), Le palais de cristal. À l'intérieur du capitalisme planétaire (traduit de l'allemand par Olivier Mannoni), Paris : Maren Sell.

SOUQUET Lionel (2002), «L'identité argentine ou la construction d'un mythe littéraire entre Europe et Amérique », Amnis, Revue électronique de civilisation contemporaine Europe/ Amériques, 2, Les identités culturelles et nationales dans les sociétés européennes et américaines, 215-244, <www.univ-brest.fr/amnis/documents/Souquet2002>.

SOUQUET Lionel (2005), « Manuel Puig : de l'écriture de la trahison à la trahison de l'écriture », Actes du colloque international d'ALMOREAL : La trahison / La traición (Angers, 2004), Centre de recherche Universités Angers-Le Mans-Orléans, 109-118.

SOUQUET Lionel (2007), « Homosexualité et révolution : Puig, Lemebel, Arenas et les “aléas" de la figure de l'homosexuel dans Fresa y chocolate », Les Langues néo-latines, 343, 165-182.

SOUQUET Lionel (2013), « Copi : l'Immoderato cantabile d'un Argentin francophone », F. Morcillo et C. Pélage (dir.), Littératures en mutation. Écrire dans une autre langue (p. 131-158), Orléans : Paradigme.

STURZENEGGER Odina (2006), L'Argentine, Paris : Karthala. 
URDICIAN Stéphanie (2006), « Texte, paratexte, hypertexte : aux carrefours du sens dans l'œuvre de Griselda Gambaro », communication présentée aux Journées d'études du séminaire Amérique latine « Le texte et ses liens » (sous la direction de M. Ezquerro), Institut d'études ibériques et latino-américaines (3-4 juin 2005), Université Paris 4, Le texte et ses liens I / El texto y sus vínculos I (p. 245-258), Cultures et littératures hispano-américaines, Les Ateliers du SAL, Paris : Indigo \& Côté-femmes éditions.

URDICIAN Stéphanie (2009), Le théâtre de Griselda Gambaro, Paris : Indigo \& Côté-femmes éditions.

WAJNMAN Solange (1994), Le kitsch et l'esprit du temps : les traces du goût populaire dans le vécu postmoderne. Étude de la « Forme » kitsch à travers l'art et la communication de masse au Brésil (thèse de doctorat sous la direction de M. Maffesoli), Université Paris Descartes.

\section{NOTES}

1. «Est-ce que la férocité, l'absurde, la violence, le désir, la chute des masques, le cannibalisme des pièces de Copi sont argentins ? Bien sûr. » (Je traduis.)

2. Comme Gilles Delavaud (2005) le montre, il existe toutes sortes de "théâtre télévisé ", mais il est fort probable que le «teleteatro» dont parle Puig se rapproche des "telenovelas» ou « culebrones» que regardent les héroïnes de Cae la noche tropical. Sur les émissions populaires de la télévision brésilienne : Wajnman (1994).

3. Peut-être s'agit-il ici d'une allusion au film de Marcel Carné, Les visiteurs du soir (1942).

4. «LE MAîTRE DE MAISON. - (S'apitoyant car il est toujours attentif à la santé mentale de la Fille, qu'il considère fragile.) Ne t'inquiète pas [...] Quand une jeune fille est déflorée c'est toujours la même chose, elle imagine que ses parents la découvrent [...] nous sommes une hallucination. C'est ta conscience coupable qui te fait avoir des visions [...].» (Je traduis.)

5. «[...] c'est elle la seule coupable, d'abord épouse adultère et maintenant meurtrière... » (Je traduis.)

6. «Le bonheur qu'elle nous donnait était trop grand, nous ne pouvions le supporter, nous seuls avons commencé à imaginer des obstacles, et de là à les matérialiser il n'a fallu qu'un pas. » (Je traduis.)

7. «S'il revient pour toi, cela signifie que tu en vaux la peine [...] (Je traduis.)

8. M. Ezquerro (1983) en donne un résumé, beaucoup plus bref, dans un article où elle analyse la pièce.

9. «[...] sont jeunes, trente ans environ, mais en même temps leur air mondain les fait paraître plus vieux, d'un âge indéterminé. » (Je traduis.)

10. «Rien n'est réaliste, tout est stylisé. » (Je traduis.)

11. «[...] Le changement de sexe par lequel passe Manuel Puig à la fin de son traité sur la sexualité (parodique, en partie du moins) montre que l'autorité peut bel et bien être questionnée et retournée, tant dans le "texte d'en bas" que dans celui “d'en haut". » (Je traduis.)

12. Lia Beatriz Lateana (2005) constate que la notion de «schizophrénie » reste cryptique pour le grand public.

13. «Le médecin déplie la camisole de force et la lui met avec amour pendant qu'ils parlent. » (Je traduis.)

14. Jorge Salessi (2000) montre aussi, dans un essai passionnant, comment, dans l'Argentine de la fin $d u x x^{e}$ et $d u$ début $d u x^{e}$ siècle, les médecins et psychiatres hygiénistes ont fortement contribué, dans le cadre d'une violente campagne d'éducation nationaliste, à «dresser » la population immigrante, tentant d'éradiquer toute forme de «perversion» - dont l'homosexualité - pour forger une Argentine «idéale » et lisse. 
15. Née au début des années 1960, l'antipsychiatrie s'oppose à la psychiatrie classique et appréhende la maladie mentale dans une perspective sociologique, relativisant les notions de «normalité » et de "pathologie». À l'instar du praticien anglais David Cooper, les antipsychiatres pensent, comme le philosophe Michel Foucault, que la psychiatrie est une institution non pas médicale, mais plutôt politique et/ou religieuse médicalisée, s'attachant à résoudre le problème de la folie - considérée comme un dysfonctionnement social - par des procédés coercitifs (internement, électrochocs, lobotomie, etc.), incompatibles avec les principes d'un État de droit.

16. «Il y a deux ans [en 1973] l'Argentine avait un des services psychiatriques, gratuits, les plus évolués du monde. Des gens de l'école anglaise de Cooper sont venus étudier le phénomène, ceux de l'antipsychiatrie, ce qu'il y a de plus avancé au monde, ainsi que des disciples de Mélanie Klein, des Français [...] Au moment de mon départ on démantelait tous les services psychiatriques des hôpitaux, les gratuits. On ne va laisser que les asiles d'aliénés [...] Le gouvernement dit que les psychiatres sont subversifs, qu'ils ont une orientation marxiste. » (Traduction de A. Bensoussan.) 17. « une luxueuse maison de campagne » (je traduis.)

18. «Ils sont élégants, mais d'une façon extrêmement sobre et opaque [...] Ce qui retient particulièrement l'attention c'est qu'elle n'ait pas teint ses cheveux blancs. Leurs vêtements correspondent aussi au cliché habituel sur la bourgeoisie rurale. » (Je traduis.)

19. «(Il reprend peu à peu son flegme et son air de supériorité habituels, derrière lesquels il cache toujours ses vrais sentiments.) » (Je traduis.)

20. Les États-Unis ayant décidé, en 1948, de ne pas étendre le plan Marshall à l'Espagne, Franco et Perón signent un protocole par lequel l'Argentine s'engage à aider économiquement l'Espagne en échange de certains avantages (Bonardi, 2004).

21. « une ambiance où le monde semble se désintégrer » (je traduis).

22. «[...] nous sommes une hallucination. » (Je traduis.) Aussi curieux que cela puisse paraître, la pièce de Puig utilise le même ressort dramatique que le chef-d'œuvre de Calderón, La vida es sueño (La Vie est un songe, 1635) puisque, dans les deux drames, le père du personnage central (Segismundo/Sigismond et Hija/La Fille) plonge volontairement sa progéniture dans une confusion entre la réalité et le rêve afin de la manipuler. Là s'arrête, probablement, la comparaison car la pièce de Calderón propose une réflexion métaphysique qui n'est pas à l'œuvre dans ce texte de Puig.

23. «[...] notre sort n'aurait pas pu être aussi triste. Elle n'a pas perdu la raison et ils ne l'ont pas emmenée à l'asile. Elle est ici, en train de jouer avec nous comme quand elle était petite » (je traduis).

24. Míguez (1999 : 21-45) montre clairement comment le modèle bourgeois s'est peu à peu imposé dans toute la classe moyenne argentine.

25. Il faut signaler que Puig cite Reich dans les notes de bas de pages de El beso... (p. 155). Il rappelle qu'Altman classe Reich parmi les « libertaires» traditionnels pour qui la libération sexuelle s'exprime dans un « orgasme parfait », obligatoirement hétérosexuel.

26. «Mais dites-moi un peu, vous n'avez pas honte de vous laisser traiter comme ça ?, ou est-ce que ce que je vous paye me donne le droit de vous maltraiter ? Répondez ! » (Je traduis.)

27. «PATIENTE. - (L'éclairage réaliste revient, la Patiente reprend sa personnalité habituelle, elle se retrouve dans la même position qu'au moment de la coupure en dehors de la réalité.) Vous ne me comprenez pas ? Pourquoi restez-vous à me regarder comme ça ?» (Je traduis.)

28. «Souvenez-vous que là-bas, ces catégories - sain et malade - n'existent pas. Ils sont tous malades. » (Je traduis.)

29. Dès le retour d'exil du général Perón, en 1973, le massacre d'Ezeiza du 20 juin marque la scission entre les péronistes de gauche (Montoneros) et la bureaucratie syndicale de droite ainsi que l'extrême droite, dont toute une partie soutient alors Perón - et que Perón soutient. José López Rega, Raúl Alberto Lastiri, Perón lui-même (1973-1974), puis sa femme Isabel (1974-1976) 
mettent alors en place la Triple A (Alliance Anticommuniste Argentine), un escadron de la mort qui fit des milliers de victimes. La répression d'État commence donc avant le coup d'État de mars 1976 qui mène la junte militaire au pouvoir et déloge Isabel Perón.

30. «Maman m'avait dit que si je ne restais pas à ma place, je ne m'attirerais que des ennuis. Aussitôt dit, aussitôt fait. » (Je traduis.)

31. Bien qu'il ne cite pas Deleuze, Aira reprend ici une notion typiquement deleuzienne : «Il n'y a pas plus de début que de fin. On arrive toujours au milieu de quelque chose, et l'on ne crée qu'au milieu en donnant de nouvelles directions ou bifurcations à des lignes préexistantes. » (Deleuze, 2003 : 199)

32. « Et peut-être que celles qu'on a oubliées serviraient bien à quelque chose... » (Je traduis.)

\section{RÉSUMÉS}

C'est en 1968 que Copi (Raúl Damonte Botana, 1939-1987) publie sa première pièce, La journée d'une rêveuse puis, un an plus tard, la très iconoclaste Eva Peron. Par son milieu familial, Copi a baigné dès l'enfance dans la vie théâtrale internationale et a été fortement influencé par sa grand-mère, la dramaturge anarcho-féministe Salvadora Medina Onrubia de Botana. Mais, vivant en exil avec sa famille, Copi est coupé de la réalité de la scène argentine et il écrit d'ailleurs ses premières pièces, comme presque toute son œuvre, en français. Cependant, pour Jorge Dubatti, il est évident que Copi reste un auteur argentin. Manuel Puig (1932-1990), romancier déjà reconnu depuis 1968, viendra au théâtre tardivement et presque par hasard, en 1978, avec l'adaptation scénique de El beso de la mujer araña (créée en 1981). Bajo un manto de estrellas, en 1981, puis la publication posthume de cinq autres pièces et comédies musicales - dont El misterio del ramo de rosas - montreront que l'écriture dramatique n'était pas un simple épiphénomène dans l'œuvre du romancier cinéphile. Le théâtre de Puig - mise en abyme de la théâtralisation œdipienne - et celui de Copi - « maniériste », « inopportun » (Une visite inopportune) et sans complaisance - sont parfaitement opératoires et subversifs. Selon une perspective souvent métathéâtrale, « mineure » et révolutionnaire (Deleuze, « Un manifeste de moins ») mais universelle (oscillant entre métaphysique et politique), et malgré une distance apparente, ces deux dramaturges argentins de l'exil interrogent profondément l'identité argentine.

In 1968, Copi (Raúl Damonte Botana, 1939-1987), published his first play, La journée d'une rêveuse followed a year later by the iconoclastic Eva Peron. Brought up in a theatrical family, Copi was surrounded by international theatre from an early age and was especially influenced by his grandmother, the anarchist-feminist playwright Salvadora Medina Onrubia de Botana. However, living in exile with his family, Copi was cut off from the Argentinian stage of the time and wrote his first plays (and most of his subsequent works) in French. Nonetheless, for Jorge Dubatti, Copi clearly remains an Argentinian writer. Manuel Puig (1932-1990), a well-known novelist by 1968, came to the theatre much later and almost by chance in 1978, with the stage adaptation of El beso de la mujer araña and Bajo un manto de estrellas in 1981. Following this period, the posthumous publication of five other plays and musicals-including El misterio del ramo de rosas-have proven that this foray into the theatre was not a mere epiphenomenon in the film-loving writer's works. Puig's theatre (a mise en abyme of oedipal theatrialisation) and that of Copi-“mannerist", "inappropriate" (Une visite inopportune) and in no way complacent-are both perfectly operative and subversive. From a frequently metacritical, "minor" and revolutionary (Deleuze, "Un 
manifeste de moins") yet universal perspective which oscillates between the metaphysical to the political, and in spite of the ostensible distance between them, both exiled Argentinian playwrights profoundly call into question Argentinian identity.

INDEX

Keywords : Copi, Manuel Puig, Argentina, theatre, psychoanalysis, politics, Deleuze (schizoanalysis)

Mots-clés : Copi, Manuel Puig, Argentine, théâtre, psychanalyse, politique, Deleuze (schizoanalyse)

\section{AUTEUR}

\section{LIONEL SOUQUET}

Université de Bretagne occidentale (Brest), E.A. 4249 HCTI 